\title{
3D MODELING OF ACCRETION SHOCKS IN YOUNG STELLAR OBJECTS: SIMULATION OF LABORATORY EXPERIMENTS
}

\author{
L. Ibgui ${ }^{1}$, M. González ${ }^{2}$, C. Stehlé ${ }^{1}$, I. Hubeny ${ }^{3}$ and T. Lanz ${ }^{4}$
}

\begin{abstract}
In order to improve the understanding of the physics of accretion shocks around young stellar objects, we have performed a three dimensional simulation of a radiative shock generated in a laser installation. We depict the 3D structure of such a shock. Radiation hydrodynamics is modeled with the HERACLES code; then, radiative transfer post-processing is performed with the IRIS code.
\end{abstract}

\section{Introduction}

Star formation is directly related to protoplanetary disks and to birth of planetary systems. It is therefore crucial to model correctly the various stages of the evolution from an interstellar cloud up to a well established planetary system. We are interested in pre-main sequence phase of stellar evolution. T Tauri stars deserve specific attention, because their masses are comparable to the solar mass, and thus may be considered as prototypes of the young Sun. According to the classic magnetospheric accretion scenario, the disk is disrupted by the stellar magnetic field and the ionized material falls onto the star along magnetic funnels, which generates a strong radiative shock on the stellar surface (Bouvier et al. 2007). Radiative shocks can also be experimentally generated by high energy laser facilities (Stehle et al. 2010). Our objective is to characterize theoretically the structure of these experimental shocks. In Section 2, we briefly present our physical models and numerical tools. In Section 3, we describe the three-dimensional structure of the simulated radiative shock.

${ }^{1}$ LERMA, Observatoire de Paris, CNRS, UMPC, 5 place J. Janssen, 92195 Meudon Cedex, France

2 AIM, CEA/DSM/IRFU, CNRS, Université Paris Diderot, 91191 Gif-sur-Yvette, France

${ }^{3}$ Department of Astronomy, Steward Observatory, The University of Arizona, 933 N. Cherry Ave, Tucson, AZ 85721-0065, USA

${ }^{4}$ Laboratoire J.-L. Lagrange, Université de Nice Sophia Antipolis, CNRS, Observatoire de la Côte d'Azur, Boulevard de l'Observatoire, BP. 4229, 06304 Nice Cedex 4, France 


\section{HERACLES and IRIS: Short description of the models and of the codes}

\subsection{HERACLES}

HERACLES is a second-order Godunov-type radiation-hydrodynamics code using the $\mathrm{M}_{1}$ radiative transfer model (González et al. 2007). We use a perfect gas equation of state for the internal specific energy (erg/g):

$$
e=\frac{P}{\rho(\gamma-1)}=(1+\langle Z\rangle) \frac{k T}{\mu m_{u}(\gamma-1)}
$$

where $\langle Z\rangle$ is the mean ionization stage, $\mu$ is the gas atomic weight, $m_{u}$ is the atomic mass unit, $P$ is the pressure, $\rho$ is the density, $T$ is the temperature, $k$ is the Boltzmann constant, and $\gamma$ is the adiabatic coefficient.

In the following applications (see Sect. 3), the gas is pure Xenon $\left(\mu_{X e}=\right.$ 131.29). In order to roughly recover the experimental compression ratio, and, as the ionization energy is neglected in our simulations, we take $\gamma=1.1$. We also assume a grey radiative transfer model, in which the Xenon opacities and ionization stages (the latter range from 0 to 8.5 in the simulations presented here) are calculated with the POTREC model (Mirone et al. 1997).

\section{$2.2 I R I S$}

IRIS is a generic three-dimensional radiative transfer code (Ibgui et al. 2013a), whose purpose is to post-process 3D (radiation) (magneto) hydrodynamics (RMHD) simulations, in order to provide spectroscopic signatures of a given astrophysical object. It solves the exact monochromatic 3D radiative transfer equation (RTE) (HERACLES uses an approximate treatment of radiative transfer, assuming the grey model or a multigroup approach). IRIS assumes local thermodynamic equilibrium (LTE), and a time-independent situation. Both these restrictions will be removed in the future.

The RTE is solved in a Cartesian grid, with cubic cells of arbitrary sizes and irregular spacing in each direction: we have $\Delta x_{\text {cell }}(x), \Delta y_{\text {cell }}(y)$, and $\Delta z_{\text {cell }}(z)$. The thermodynamic properties (temperature, density, velocity) are determined from the RMHD simulations. The opacities are defined as input data for the code, at each grid point and for a grid of frequencies. The specific intensity is calculated as a function of frequency, position, and direction, with the short-characteristics method (Kunasz \& Auer 1988). Interpolations are performed with Bezier cubic polynomials (Auer 2003). This approach efficiently reduces the numerical diffusion effect of the short-characteristics method. Boundary conditions may be specified by the user. Alternatively, for a horizontally periodic infinite medium, boundary conditions are self-consistently determined by the code (Ibgui et al. 2013a). The code provides, as functions of the frequency $\nu$ and the position $(x, y, z)$, the 
following properties of the radiation field: the mean intensity $J$, the radiation flux vector $F_{x}, F_{y}, F_{z}$, and the radiation pressure tensor $P_{x x}, P_{y y}, P_{z z}, P_{x y}, P_{x z}, P_{y z}$.

\section{3D structure and radiative properties of a radiative shock}

Laboratory radiative shock experiments were performed at the high-energy laser PALS facility (Prague, Czech Republic) (Stehle et al. 2010; Stehle et al. 2012). The laser pulse is focused on a foil ("piston") that closes a millimetric scale shock tube. The laser energy is converted into mechanical energy (Stehle et al. 2009). Prior simulations with HERACLES had revealed multidimensional effects in such shocks, such as lateral radiative losses (González et al. 2006; González et al. 2009). In order to characterize the radiative structure and spectroscopic signatures of these shocks, we use IRIS to post-process the results from HERACLES.

We simulate here a radiative shock in a $6 \mathrm{~mm}$ long shock tube full of pure Xenon, with a $0.4 \times 0.4 \mathrm{~mm}^{2}$ uniform section (see Fig. 1). In order to reproduce the experimental conditions described by Stehle et al. (2012), we assume that the piston moves with a constant velocity of $60 \mathrm{~km} \mathrm{~s}^{-1}$ relative to the laboratory frame (in positive $z$-direction in the coordinate system shown in Fig. 1). The unshocked gas in the far upstream flow is immobile relative to the laboratory frame. It has a density of $10^{-3} \mathrm{~g} \mathrm{~cm}^{-3}$, and a temperature of $1 \mathrm{eV}$. The shock, generated by the moving piston, propagates in positive $z$-direction with a velocity that evolves before reaching a stationary limit (González et al. 2009). We show here a snapshot of the radiative shock evolution at a time when the shock is far enough from the piston (10 ns after the launching of the piston), but not necessary at the stationary limit. Hydrodynamics simulations show that the shocked gas is immobile relative to the piston. As for the radiation, we adopt the following boundary conditions for each of the six walls of the tube. The base of the tube, which models the piston, emits like a black body whose local temperature equals the local temperature of the neighboring gas. The four lateral tube walls have a zero albedo, which means that there is no wall reflection, i.e., the photons can freely escape from the walls. In the same vein, the photons can freely travel through the right face of the tube.

In this study, we focus on the radiation flux in $z$-direction, $F_{z}(x, y, z)$ (assuming a grey model). The determination of the lateral fluxes $F_{x}, F_{y}$ is in progress. We will present the results in a forthcoming paper (Ibgui et al. 2013b). Figure 2 displays the four following quantities along $z$-axis, at the center of the cross section of the tube, i.e., at $(x, y)=\left(x_{\text {center }}, y_{\text {center }}\right)=(0.2,0.2) \mathrm{mm}$ : radiation flux $F_{z}$, temperature $T$, grey opacity $\kappa$, and density $\rho$. We note a quite good agreement between the flux calculated by HERACLES (approximate model) and the flux calculated by IRIS (exact RTE solved). The shock front is at the temperature peak, $z_{\text {front }}=0.033 \mathrm{~mm}$. Once heated by the shock, the plasma restitutes a fraction of the gained energy by emitting photons in all directions. As the flux profile in Figure 2 shows, photons emitted in the $z$-direction travel much larger distances in the unshocked gas $\left(z>z_{\text {front }}\right)$ than in the shocked gas $\left(z<z_{\text {front }}\right)$. This discrepancy between the distances is explained by the opacity of the gas, which 


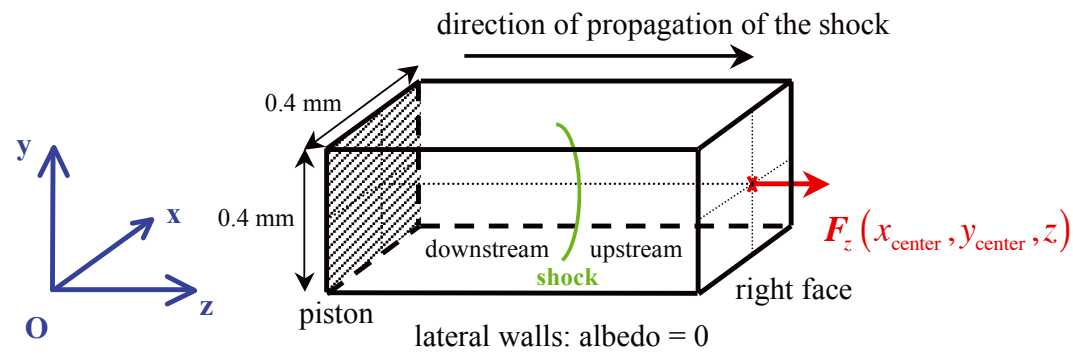

Fig. 1. Tube, full of pure Xenon, in which the radiative shock is generated. The piston moves with a constant velocity of $60 \mathrm{~km} \mathrm{~s}^{-1}$ relative to the laboratory frame (in positive $z$-direction in the coordinate system shown here). Far upstream from the shock, the fluid is immobile relative to the laboratory frame, its density is $10^{-3} \mathrm{~g} \mathrm{~cm}^{-3}$, its temperature is $1 \mathrm{eV}$. The photons can freely escape from the lateral walls for which a zero albedo is assumed. $F_{z}\left(x_{\text {center }}, y_{\text {center }}, z\right)$ is the radiation flux in $z$-direction, in the center of the cross section of the tube, at $z$, where $z=0$ refers to the position of the piston.

significantly increases in the shocked part. The temperature immediately ahead of the shock is close to the temperature behind it. In addition, the flux profile is asymmetric, and the temperature profile reveals an extended radiative precursor. These features are characteristic of a supercritical shock (Zel'dovich \& Raizer 1967; Mihalas \& Mihalas 1984; Ensman 1994). The flux $F_{z}$ can become slightly negative in the shocked medium, around $z=0.02 \mathrm{~mm}$. This was already predicted in onedimensional simulations of radiative shocks (Ensman 1994; Gehmeyr \& Mihalas 1994; Sincell et al. 1999; Sekora \& Stone 2010).

The three-dimensional nature of the radiative shock is revealed by Figure 3, which represents the distribution of the flux $F_{z}$ and the temperature $T$ over the cross section $(x, y)$ at a given position $z$ in the tube. We choose a position located in the radiative precursor, at $z=0.066 \mathrm{~mm}$, which is indicated by large dots in Figure 2. The radiation flux $F_{z}$ has a curved shape, with an amplitude that decreases near the borders of the $(x, y)$ surface. Such radiative effects have a direct impact on the structure of the flow since the temperature distribution over the $(x, y)$ surface is analogous to the flux $F_{z}$ distribution. We are currently investigating the evolution of these quantites in the shocked part of the flow. We plan to show the results in our forthcoming paper.

\section{Summary}

In order to improve our understanding of accretion shocks in young stellar objects, we have simulated the hydrodynamic and radiative properties of a radiative shock generated in laboratory. We have highlighted the three-dimensional nature of such a flow, by showing the curved shapes of the flux and the temperature in a cross section of the flow, in the radiative precursor zone. 

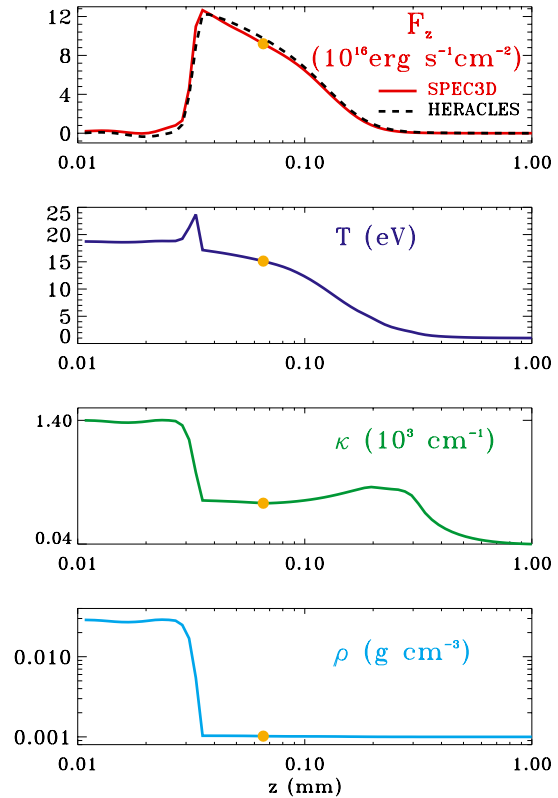

Fig. 2. Radiation flux $F_{z}$, temperature $T$, opacity $\kappa$, and density $\rho$ versus $z$, at the center $\left(x_{\text {center }}, y_{\text {center }}\right)$ of the cross section of the tube. $\kappa$ and $\rho$ are plotted with logarithmic scales. The agreement is good between $F_{z}$ calculated by HERACLES (approximate model) and $F_{z}$ calculated by IRIS (exact RTE solved). The radiative precursor is clearly seen in the temperature profile. Thick dots indicate a $z$-position at which maps of the flux and temperature are plotted versus $(x, y)$ in the cross section (see Fig. 3 ).
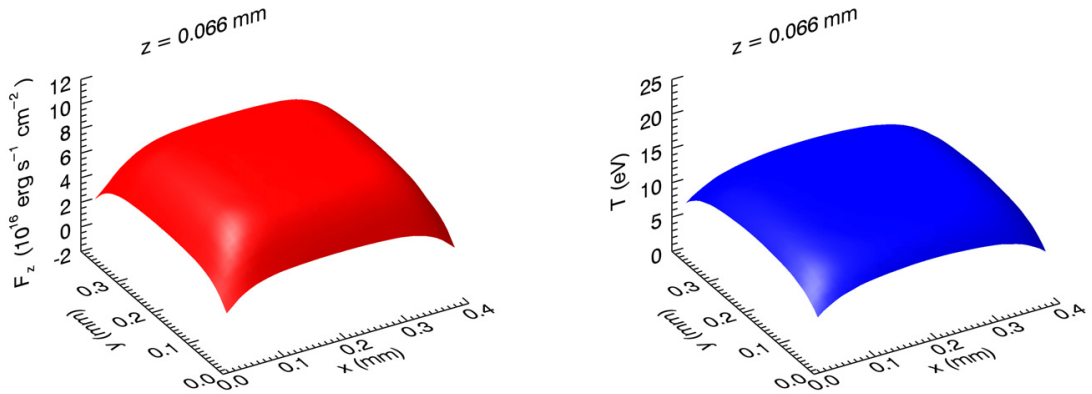

Fig. 3. Radiation flux calculated by IRIS, $F_{z}\left(10^{16} \mathrm{erg} \mathrm{s}^{-1} \mathrm{~cm}^{-2}\right)$ (left), and temperature $T(\mathrm{eV})$ (right) at a cross section of the tube, functions of $(x, y)$, and for a given position $z=0.066 \mathrm{~mm}$.

Further investigations include the quantitative study of lateral fluxes, $F_{x}$ and $F_{y}$, along with a detailed analysis of the shocked medium. Such a work is in progress. 
The work is supported by the French ANR, under grant 08-BLAN-0263-07. IH acknowledges the support of Observatoire de Paris.

\section{References}

Auer, L., 2003, ASP Conf. Ser., 288, 3

Bouvier, J., Alencar, S.H.P., Harries, T.J., Johns-Krull, C.M., \& Romanova, M.M., 2007, Protostars and Planets V, 479

Ensman, L., 1994, ApJ, 424, 275

Gehmeyr, M., \& Mihalas, D., 1994, Physical D 77, 320

González, M., Stehlé, C., Audit, E., Busquet, M., et al., 2006, A\&A, 464, 429

González, M., Audit, E., \& Huynh, P., 2007, A\&A, 464, 429

González, M., Audit, E., \& Stehlé, C., 2009, A\&A, 497, 27

Ibgui, L., Hubeny, I., Lanz, T., \& Stehlé, C., 2013a, A\&A, 549, A126

Ibgui, L., González, M., Stehlé, C., Lanz, T., Hubeny, I., \& Chièze, J.-P., 2013b, in preparation

Kunasz, P., \& Auer, L.H., 1988, JQSRT, 39, 67

Mihalas, D., Weibel-Mihalas, B., 1984, Foundations of Radiation Hydrodynamics (Oxford University Press)

Mirone, A., Gauthier, J.C., Gilleorn, F., \& Chenais-Popovics, C., 1997, JQSRT, 58, 791

Sekora, M.D., \& Stone, J.M., 2010, J. Comput. Phys., 229, 6819

Sincell, M.W., Gehmeyr, D., \& Mihalas, D., 1999, Shock Waves, 9, 403

Stehlé, C., González, M., Audit, E., \& Lanz, T., 2009, ed. K. Tsinganos et al., Protostellar Jets in Context, 359

Stehlé, C., González, M., Kozlova, M., et al., 2010, Laser Part. Beams, 28, 253

Stehlé, C., Kozlová, M., Larour, J., et al., 2012, Opt. Communications, 285, 64

Zel'dovich, Y.B., \& Raizer, Y.P., 1967, Physics of Shock Waves and High Temperature Hydrodynamic Phenomena (Academic Press, New York) 